\title{
NISTIR 6738
}

\section{Proposed Diffuse Ambient Contrast Measurement Methods for Flat Panel Displays}

\section{Edward F. Kelley}

U.S. DEPARTMENT OF COMMERCE

Technology Administratıon

Electricity Division

Electronics and electrical Engineering Laboratory

National Institute of Standards and Technology

Gaithersburg, MD 20899

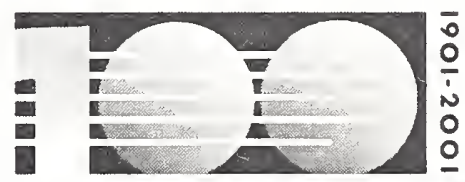

NIST CENTENNIALE

QC

100

NLT

National Institute of

$-456$

Standards and Technology

No. 4738

Technology Administration

U.S. Department of Commerce 



\section{Proposed Diffuse Ambient Contrast Measurement Methods for Flat Panel Displays}

\section{Edward F. Kelley}

U.S. DEPARTMENT OF COMMERCE Technology Admınıstratıon

Electricity Division

Electronics and electrical Engineerıng Laboratory

National Institute of Standards and Technology

Gaithersburg, MD 20899

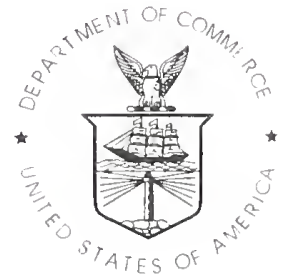





\section{DIFFUSE AMBIENT CONTRAST MEASUREMENTS (CONTRAST WITH REFLECTIONS UNDER DIFFUSE ILLUMINATION)}

We want to measure the contrast (ratio of white to black) of a display under uniform diffuse illumination to provide an indication of the legibility of the display under diffuse-ambient lighting conditions. ${ }^{1}$ This is the type of illumination that can be generated by a bright overcast day. Several methods can be used to make this kind of measurement. The methods vary depending upon the way the diffuse illumination is provided. We show three methods that should provide ambient contrast measurement results with an uncertainty of no greater than $6 \%$ (expanded uncertainty with a coverage factor of two-in the past described as a two-sigma uncertainty). These are the (1) Integrating Sphere Method, (2) Open Box Method, and (3) Sampling Sphere Method. We will describe how the measurement results are properly scaled, discuss the tight sources to be used, obtain the starting conditions in Step 0, and detail the methods. These methods can be applied to both emissive and reflective displays. In the following, we show a flat panel display (FPD) being measured, but these results are applicable to all direct-view displays.

Scaling for Typicall Illuminance: In all these mcasurements, we want to characterize the display for a typical diffuse lighting environment in which the display will be used. For example, for use outside we might want to measure the contrast of the display under a diffuse illuminance of $E_{0}=6000 \mathrm{~lx}$. Providing that exact illuminance may be difficult in practice, so we resort to scaling. All the measurements will therefore be scaled for some selected ambient iltuminance $E_{0}$ unless that illuminance can be precisely provided dircetly.

Lamp Sources: The light sources or lamps used for all these reflection measurements should be $2856 \mathrm{~K}$ tungsten-halogen lamps or similar broadband illumination. If another source is used or required, it should be specified clearly with the reported results. The spectral content of the source can substantially affect the measured reflectance results. Whereas a D65 illuminant is a better simulation of daylight conditions, D65 illumination is often difficult to provide accurately. Often such lamps that are claimed to be D65 simply achieve the D65 white point, but their spectrum may be significantly different from the D65 spectrum specified by the CIE (Commission Internationale de l'Eclairage [International Commission on lllumination]). It is for the reason of reproducibility and convenience that we specify the CIE Illuminant A (2856 K tungsten-halogen source) for these measurements.

STEP 0: Many types of displays need to be properly warmed up prior to measurement to assure their electronics and backlight (if used) are stable. Before an emissive display is placed
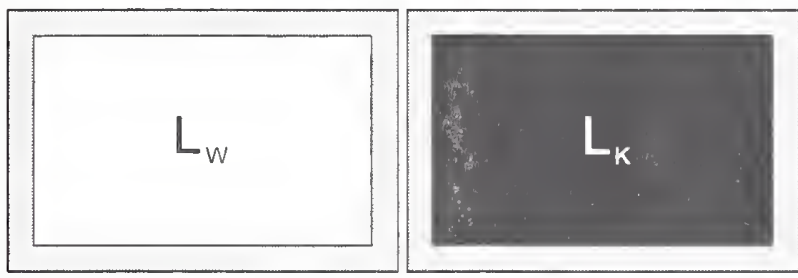

Fig. 1. Intrinsic full-screen white and black huminance measurements are made before the reflection measurements are implemented (for emissive displars). within the reflection measurement enclosures or before the sampling sphere is placed over the display, a measurement must be made of the luminance of the display for full-screen white $L_{W}$ and for full-screen black $L_{\mathrm{K}}$ using a luminance meter-see Fig. 1. The measurement should be made in a darkroom environment so that no reflections arc introduced from the environment including the operator and the instrumentation. If a darkroom is not available or the display cannot be removed from its environment, a darkroom environment can be fashioned from opaque black cloth placed around the display and luminance meter to eliminate any light from the surround, or some similar arrangement can be implemented to etiminate stray light. NOTE: In the case of reflective displays (non-emissive) there is generally no need to make darkroom measurements on the display for diffuse ambient performance measurements because $L_{W}$ and $L_{\mathrm{K}}$ are both zero, i.e., $L_{W}=L_{\mathrm{K}}=0$ in the calculations below; however, the display may still need to be warmed up.

(a) Warm-up: The display should be properly warmed up (if needed) before any measurements are made. Depending upon the display, warm-up could be from $15 \mathrm{~min}$ to 1 hour or more. Often people look for a warm-up period that is long enough so that the changes in the luminances of white and black are significantly less than $1 \%$ over the measurement time period. How warm-up is defined is beyond the scope of the procedures outlined here. The required warm-up time can depend upon the requirements of the user, the application of the display, and manufacturer's specifications.

(b) Measurement of White Luminance $L_{\mathbb{W}}$ : Measure the luminance $L_{W}$ of full-screen white. If there is a significant difference (over a few percent) between the luminance at scrcen normal and at an angle of $8^{\circ} \leq \theta \leq 10^{\circ}$, then make the luminance measurement at an angle $8^{\circ} \leq \theta \leq 10^{\circ}$ to the left of normal. 
(c) Measurement of Black Luminance $L_{\mathrm{K}}$ : Measure the luminance $L_{\mathrm{K}}$ of full-screen black. If there is a significant difference (over a few percent) between the luminance at screen normal and at an angle of $8^{\circ} \leq \theta \leq 10^{\circ}$, then make the luminance measurement at an angle $8^{\circ} \leq \theta \leq 10^{\circ}$ to the left of normal.

(d) Repeat After Reflection Measurements: It is instructive to repeat the above measurements of fullscreen white and full-screen black after the reflection measurements are made in order to monitor the drift in the emissive FPD characteristics. Substantial changes (over $5 \%$ ) may indicate a problem in the display or the fact that an insufficient warm-up time was used.

\section{INTEGRATING SPHERE METHOD:}

Figure 2 shows a large

integrating sphcre enclosing the FPD in cutaway. A baffled lamp behind the display illuminates the interior of the integrating sphere. This method can yield the most reproducible results, but is often inconvenient to implement (requiring the removal of the display from its environment) or too expensive because of the cost of such a large integrating sphcre. The center of the FPD
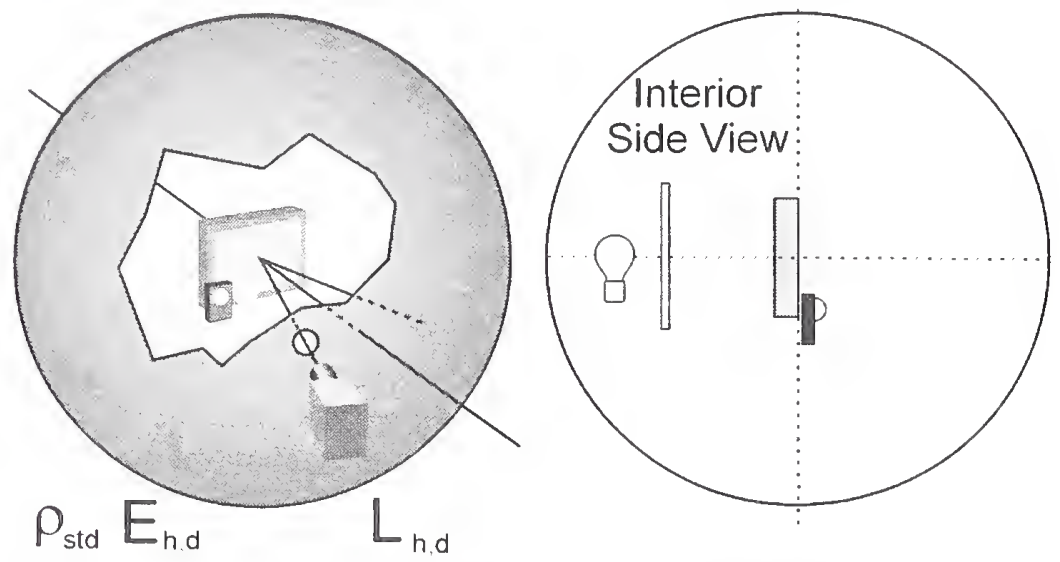

Fig. 2. Integrating sphere enclosing the FPD with illnminance meter and white diffuse standard. A baffled lamp is located behind the FPD.

screen is placed at the center of a large intcgrating sphere. The normal of the FPD is turned an angle of $8^{\circ} \leq \theta \leq 10^{\circ}$ with respect to the normal of the measurement port. Either a calibrated white-diffuse-reflectance standard of known diffuse reflectance $\rho_{\text {std }}$ or an illuminance meter (or both) is provided near the FPD surface to measure the illuminance upon the display. Avoid placing the standard or illuminance meter (or both) too close to the center of the screen so they don't interfere with the measurement of reflected luminance by obscuring some of the incident light in the measurement region. A good guideline is to place them from five to ten thicknesses (of the illuminance meter or white standard) away from the center of the FPD. The illuminance and luminance are measured for fullscreen white $\left(E_{\mathrm{l}}, L_{\mathrm{h}}\right)$ and full-screen black $\left(E_{\mathrm{d}}, L_{\mathrm{d}}\right)$. In making the luminance measurement, be sure to be far enough back from the measurement exit port so that the bright-white walls do not shine on the lens of the luminance meter. If the diffuse reflectance standard is used to provide the illuminance measurement, the illuminance $E$ is related to the luminance $L$ of the standard via

$$
E=\pi L / \rho_{\text {std }}
$$

for each screen color or mode (white or black). The procedure is as follows:

STEP 1: Measure illuminance $E_{\mathrm{h}}$ falling upon the screen and resulting luminance $L_{\mathrm{h}}$ of the screen for fullscreen white. Be sure to measure the illuminance $E_{\mathrm{h}}$ while the screen is displaying white. If an illuminance meter is not available and a white reflectance standard is used, calculate the illuminance based upon Eq. (1).

STEP 2: Calculate the diffuse reflectance ${ }^{2} \rho_{W}$ for full-screen white mode:

$$
\rho_{W}=\pi\left(L_{\mathrm{h}}-L_{W}\right) / E_{\mathrm{h}} .
$$

STEP 3: Measure illuminance $E_{\mathrm{d}}$ falling upon the screen and resulting luminance $L_{\mathrm{d}}$ of the screen for fullscreen black. Be sure to measure the illuminance $E_{\mathrm{d}}$ while the screen is displaying black. If an illuminance meter is not available and a white reflectance standard is used, calculate the illuminance based upon Eq. (1).

STEP 4: Calculate the diffuse reflectance $\rho_{\mathrm{K}}$ for full-screen black mode:

$$
\rho_{\mathrm{K}}=\pi\left(L_{\mathrm{d}}-L_{\mathrm{K}}\right) / E_{\mathrm{d}} \text {. }
$$

STEP 5: Calculate the ambient contrast $C_{\mathrm{d}}$ scaled for a diffuse illuminance of $E_{0}$ :

$$
C_{\mathrm{d}}=\frac{\left(\frac{\rho_{\mathrm{W}} E_{0}}{\pi}+L_{\mathrm{W}}\right)}{\left(\frac{\rho_{\mathrm{K}} E_{0}}{\pi}+L_{\mathrm{K}}\right)} .
$$




\section{OPEN BOX METHOD:}

Figure 3 shows a cutaway open box having a white interior and lamps positioned behind the surface of the FPD to provide as even illumination of the box interior as possible. Some refer to this as the picnic-cooler method because white closed-cell-polystyrene-foam picnic coolers have been successfully used for the box. While such a method is inexpensive, it can decrease reproducibility because of the unevenness of the luminance of the interior surface of the box. Whatever box configuration (small, large, open or enclosed) is used, two important features must be assured: (1) that the interior of the box surrounding the display has as uniform luminance as possible, and (2) direct rays from the lamps cannot hit the surface of the display.

The center of the screen of the FPD is placcd at the

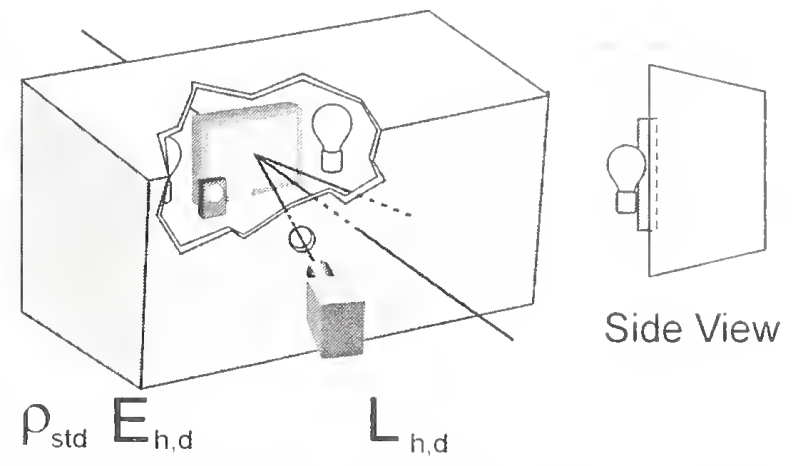

Fig. 3. Open box with white interior and lamps behind the plane of the FPD surface. center of an open box with a white interior. The screen may be placed slightly inside the plane of the box opening. A hole is provided in the back of the box for luminance measurements at an angle of $8^{\circ} \leq \theta \leq 10^{\circ}$ with respect to the normal of the screen and open surface. Two lamps are provided, one on each side of the FPD, to illuminate the box interior. (If measuring a laptop computer, it may be helpful to wrap the keyboard with white cloth should it be necessary to place the keyboard within the box.) Use lamps that provide diffuse illumination and are not directional (e.g., frosted incandescent light bulbs exhibit suitable diffusion of light whereas spotlights are too directional). The lamps should be placed behind the plane of the surface of the FPD so that no direct illumination falls on the screen from the lamps - use shielding if necessary should the back or bezel of the display not be opaque. Avoid heating the FPD by the lamps and thereby changing the FPD's characteristics. Position the lamps to provide the most uniform luminance of the box interior (this is an attempt to simulate an integrating sphere). Avoid placing the standard or illuminance meter (or both) too close to the center of the screen so that they don't interfere with the measurement of reflected luminance by obscuring some of the incident light in the measurement region. A good guideline is to place them from five to ten thicknesses (of the illuminance meter or white standard) away from the center of the FPD. Be careful that the direct rays from the lamps do not hit the illuminance meter detection surface so that a false illuminance measurement is made; a small baffle may be needed to provide the required shading. To determine the ambient contrast $C_{\mathrm{d}}$, follow the same procedure as in steps 1-5 in the above Integrating Sphere Method, Method 1.

\section{SAMPLING SPHERE METHOD:}

Figure 4 shows a $100 \mathrm{~mm}$ diameter sampling sphere that can be placed upon the surface of the FPD. ${ }^{3}$ If such a sphere is properly baffled, it can provide reproducible results comparable with the large integrating sphere of Method 1. The sampling sphere is placed upon the center of the FPD. A hole through which the luminance is measured - the measurement port-is provided in the sampling sphere at approximately an angle $\theta=10^{\circ}$ with respect to the normal of the screen and sampling port area. The measurement port and sampling port must be large enough to make a good luminance measurement. Figure 4 illustrates a sampling port and measurement port with internal diameters of $25 \mathrm{~mm}$. The minimum diameter should be approximately $20 \%$ larger than the measurement area diameter of the luminance meter. The measurement port must be of sufficient diameter so that no vignette is produced by the measurement port in the luminance-meter optics (all the rays from the measured spot must reach all parts of the lens of

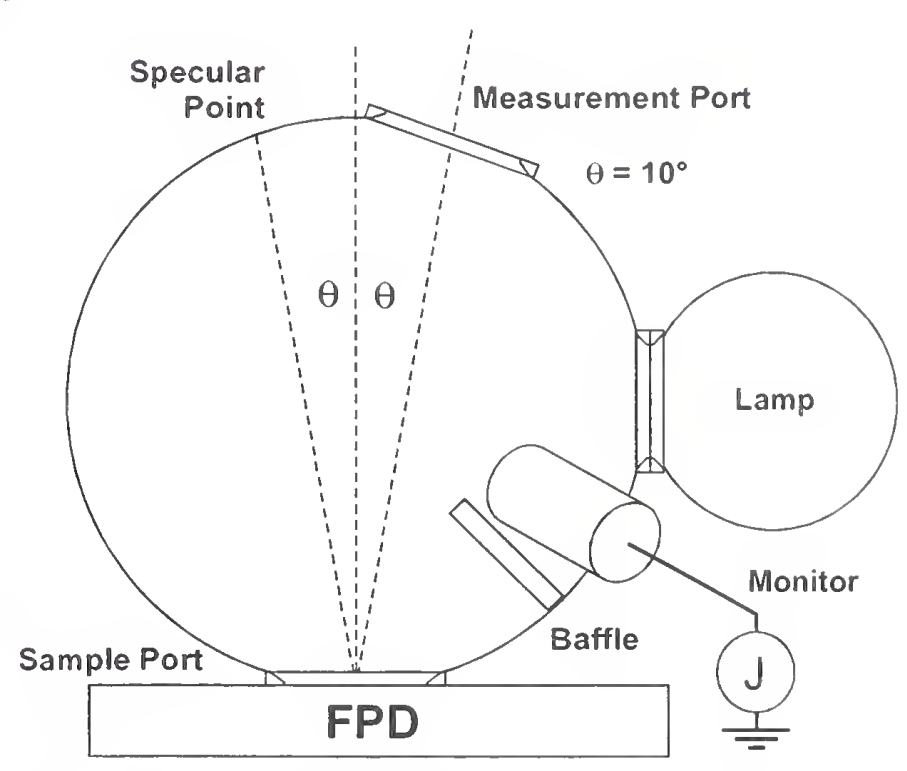

Fig. 4. Partial cross-section drawing of illuminated sampling sphere with photopic photodiode monitor. 
the luminance meter without being occulted by the measurement port). The luminance meter should be placed back far enough so its reading is not influenced by the bright internal area surrounding the sample port that can introduce glare into the measurement. A baffle is provided between the sampling port and the lamp source so that no direct rays hit the FPD surface. The photopic photodiode monitor views the interior surface away from the lamp source. A baffle may have to be placed between the photodiode monitor and the source as well as between the photodiode and the sample port so that no direct rays from either the lamp source or the FPD hit the photopic photodiode. (The need for such baffles will depend upon the construction of the photodiode monitor.) An alternative to the satellite-sphere lamp source is a fiber-optic source. The larger the sphere the more accuracy might be anticipated since the ports are likely to provide less of a perturbation on the measurement result for a larger sphere.

It may be possible to substitute a box, polyhedron, or geodesic sphere for an integrating sphere with various degrees of success depending upon the construction techniques employed. Any improvisation along these lines should be carefully tested and compared with the results using an integrating sphere as in Method I before it can be trusted. In all cases, attention must be paid to proper baffling of the sample port and the photodiode monitor. If the walls of the material used have a substantial thickness (thicker than $5 \%$ of the sampling port diameter) then attention must be given to properly beveling the hole so that the beveled ring around the sampling port is well illuminated by the interior (see cross-section of beveled port rings in Fig. 4).

Be careful in touching the FPD surface with the sampling port. Some FPDs will exhibit a change in performance with even a slight pressure applied to their surfaces. It may be necessary to carefully arrange for the sampling port to not touch the FPD surface yet be within a millimeter or so of the FPD surface.

Some FPDs, particularly those that are measured in situ, may have glass or plastic covering plates to protect the display. In such cases, the pixel surface of the FPD may be so far away from the front surface upon which the sampling sphere is placed that this sampling sphere method may not produce reliable results. In such cases, either Method 1 or Method 2 should be employed.

The photodiode monitor current $J$ must be calibrated to reflect the illuminance at the sampling port. Such a calibration can be performed in either of two ways:
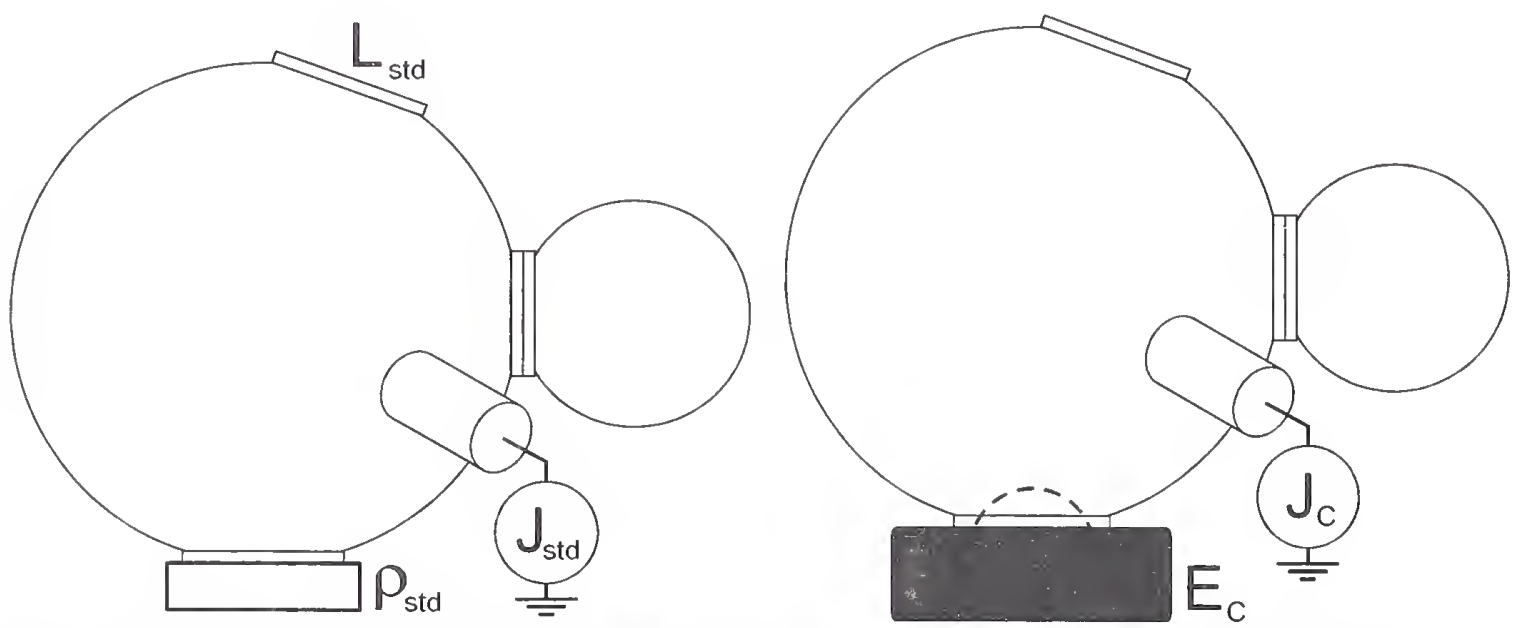

Fig. 5. Calibration of photopic photodiode monitor used with the sampling sphere is based npon either a known white reflectance standard or an illnminance meter or both.

STEP 1: Calibration of Photopic Photodiode Monitor (see Fig. 5):

(a) Using white reflectance standard: The sampling port is placed upon a white reflectance standard of known diffuse reflectance $\rho_{\text {std }}$, and the luminance $L_{\text {std }}$ and photodiode current $J_{\text {std }}$ are recorded. The illuminance $E_{\text {std }}$ is related to the luminance via

$$
E_{\text {std }}=\pi L_{\text {std }} / \rho_{\text {std }},
$$

and the calibration constant $\alpha$ is given by

$$
\alpha=E_{\text {std }} / J_{\text {std }} \text {. }
$$

The illuminance $E$ for any other configuration can be obtained by measuring the photodiode current $J$ via

$$
E=\alpha J .
$$


(b) Using an illuminance meter: The sampling port is placed upon an illuminance meter and the photodiode current $J_{\mathrm{c}}$ is recorded along with the illuminance $E_{\mathrm{c}}$. The calibration constant is given by

$$
\alpha=E_{\mathrm{c}} / J_{\mathrm{c}} \text {. }
$$

Note: If both a white diffuse standard and an illuminance meter are available, it is instructive to comparc the values of $\alpha$ obtained by both methods of calibration. The overall uncertainty in the mcasurcment using the sampling sphere is reflected in the uncertainty in a comparison of the $\alpha$ values.

STEP 2: Measure photocurrent $J_{h}$ from the photodiode monitor and resulting luminance $L_{h}$ of the screcn for full-screen white. (See Fig. 6)

STEP 3: Calculate the diffuse reflectance $\rho_{\mathrm{W}}$ for fullscreen white mode:

$$
\rho_{\mathrm{W}}=\pi\left(L_{\mathrm{h}}-L_{\mathrm{W}}\right) / \alpha_{\mathrm{h}} \mathrm{h}_{\mathrm{h}} \text {. }
$$

STEP 4: Measure photocurrent $J_{\mathrm{d}}$ from the photodiode and resulting luminance $L_{\mathrm{d}}$ of the screen for fullscreen black.

STEP 5: Calculate the diffuse reflectance $\rho_{\mathrm{K}}$ for fullscreen black mode:

$$
\rho_{\mathrm{K}}=\pi\left(L_{\mathrm{d}}-L_{\mathrm{K}}\right) / \alpha_{\mathrm{d}} J_{\mathrm{d}} \text {. }
$$

STEP 6: Calculate the ambient contrast $C_{d}$ scaled for a diffuse illuminance of $E_{0}$ using Eq. (4) above (repeated here).

$$
C_{\mathrm{d}}=\frac{\left(\begin{array}{c}
\rho_{\mathrm{W}} E_{0}+L_{\mathrm{W}} \\
\pi
\end{array}\right)}{\left(\begin{array}{c}
\rho_{\mathrm{K}} E_{0} \\
\pi
\end{array} L_{\mathrm{K}}\right)}
$$

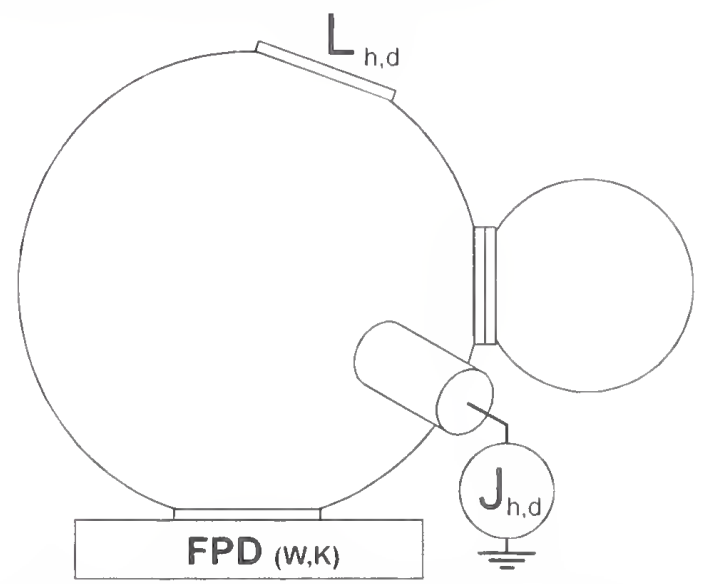

Fig. 6. Sampling sphere measurement of display surface.

\section{SAMPLE MEASUREMENT RESULTS}

The following example should not be regarded as an acceptance criterion or a goal that one would want to meet - in fact, the display employed is probably too dim for practical automotive or outdoor use. It is merely presented to illustrate how well the three measurement methods can compare even when done rather sloppily. The results obtained are for illustration purposes only and do not necessarily constitute typical or even desirable rcsults for the displays used.

The illuminance meter and luminance meter agrce to within $0.2^{\circ}{ }^{\circ}$ using an integrating sphere sourcc at a known distance. Since ratios of illuminance and luminance are especially important in these measurements, the absolute calibration of the meters is less important. Other than the agreement between meters, no particular care is used in obtaining these data. We attempt to use the same kind of attention to detail that might be characteristic of technicians in an industrial environment where speed might be preferred over meticulousness. For example, the diffuse white standard is assumed to have a diffuse reflectance of 0.99 despite having been in the open air for a number of weeks after having last been refurbished. All angles - establishment of normal, alignment of $8^{\circ}$ or $10^{\circ}$ off normal, etc.- -are approximate with an uncertainty of $2^{\circ}$ or more. Despite setups that are less than tightly controlled, the methods are found to exhibit a $3^{\circ}$ or relative standard deviation in establishing an ambient contrast measurement result. The methods are therefore judged sufficiently robust to be practical for quick and less-than-careful deployment.

The integrating sphere used in Method $\mathrm{I}$ is approximately $2 \mathrm{~m}$ in diameter. The light coming from the display when exhibiting full-screen white is negligible as seen in the fact that the illuminance on the display did not change when the screen went from white to black. Such is not the case with the open box used in Method 2, where the output from the screen has a measurable contribution to the illuminance on the screen because of the lower illuminance and volume of the box. Two common $75 \mathrm{~W}$ light incandescent bulbs are used for illumination of the box interior. The sampling sphere used in Method 3 is a dodecahedron made from closed-cell polystyrene foam plates $25 \mathrm{~mm}$ in thickness having an interior edge of the plate of $68 \mathrm{~mm}$ (it is roughly equivalent to a $125 \mathrm{~mm}$ diameter integrating sphere). The measurement port is $25 \mathrm{~mm}$ in diameter, and the sampling port is $50 \mathrm{~mm}$ in diameter. A fiber-optic illuminator is used as a source providing a slight greenish hue to the light. Therc is a measurable but small contribution of the screen flux to the illuminance on the screen because of the relatively low flux existing within the sampling sphere. No attempt is made to match color temperatures of the sources (uncertainty 
of color temperature measurement is estimated to be $30 \mathrm{~K}$ ) to CIE llluminant A. The sources are all used as is. Table 1 shows the measurement results for the reflectances $\rho_{\mathrm{W}}, \rho_{\mathrm{K}}$, and ambient contrast $C_{\mathrm{d}}$. Table 2 provides the raw data and intermediate calculations.

The reflectances exhibit a relative standard deviation of $5 \%$, but since the ambient contrast employs those reflectances in a quasi-ratio manner, the resulting relative combined standard uncertainty of the ambient contrast is seen to be $3.1 \%$. Thus, these methods-even when done hurriedly-can be expected to produce results with a relative expanded uncertainty of $6 \%$ using a coverage factor of two.

\begin{tabular}{|c|c|c|c|c|c|c|c|c|}
\hline \multirow[b]{4}{*}{ Quantity } & \multicolumn{8}{|c|}{$\begin{array}{c}\text { Table 1. Reflectances } \rho_{\mathrm{W}}, \rho_{\mathrm{K}} \text {, and Ambient Contrast } C_{\mathrm{d}} \\
\text { Based Upon Ambient llluminance } E_{0}=6000 \mathrm{~lx}\end{array}$} \\
\hline & \multicolumn{2}{|c|}{$\begin{array}{l}\text { 1. Integrating Sphere } \\
\text { Method }\end{array}$} & \multicolumn{2}{|c|}{ 2. Open Box Method } & \multicolumn{2}{|c|}{$\begin{array}{l}\text { 3. Sampling Sphere } \\
\text { Method }\end{array}$} & \multirow[b]{3}{*}{ Average } & \multirow[b]{3}{*}{$\begin{array}{l}\text { Relative } \\
\text { Standard } \\
\text { Deviation }\end{array}$} \\
\hline & \multicolumn{2}{|c|}{2954 K Source } & \multicolumn{2}{|c|}{$2589 \mathrm{~K}$ Source } & \multicolumn{2}{|c|}{$2890 \mathrm{~K}$ Source } & & \\
\hline & $\begin{array}{c}\text { llluminance } \\
\text { from } \\
\text { Measurement }\end{array}$ & $\begin{array}{l}\text { Illuminance } \\
\text { from White } \\
\text { Standard } \\
\end{array}$ & $\begin{array}{l}\begin{array}{l}\text { Illuminance } \\
\text { from } \\
\text { Measurement }\end{array} \\
\end{array}$ & $\begin{array}{l}\text { Illuminance } \\
\text { from White } \\
\text { Standard }\end{array}$ & $\begin{array}{c}\text { Calibration } \\
\text { via } \\
\text { Illuminance }\end{array}$ & $\begin{array}{c}\text { Calibration } \\
\text { via White } \\
\text { Standard } \\
\end{array}$ & & \\
\hline$\rho_{W}$ & 0.155 & 0.156 & 0.139 & 0.158 & 0.148 & 0.143 & $0 . \mathrm{I} 50$ & $5.2 \%$ \\
\hline$\rho_{\mathrm{K}}$ & 0.153 & 0.154 & 0.138 & 0.146 & 0.151 & 0.146 & $0 . \mathrm{I} 48$ & $4.0 \%$ \\
\hline$C_{\mathrm{d}}$ & 1.30 & 1.30 & 1.33 & 1.39 & 1.27 & 1.28 & 1.31 & $3.1 \%$ \\
\hline
\end{tabular}

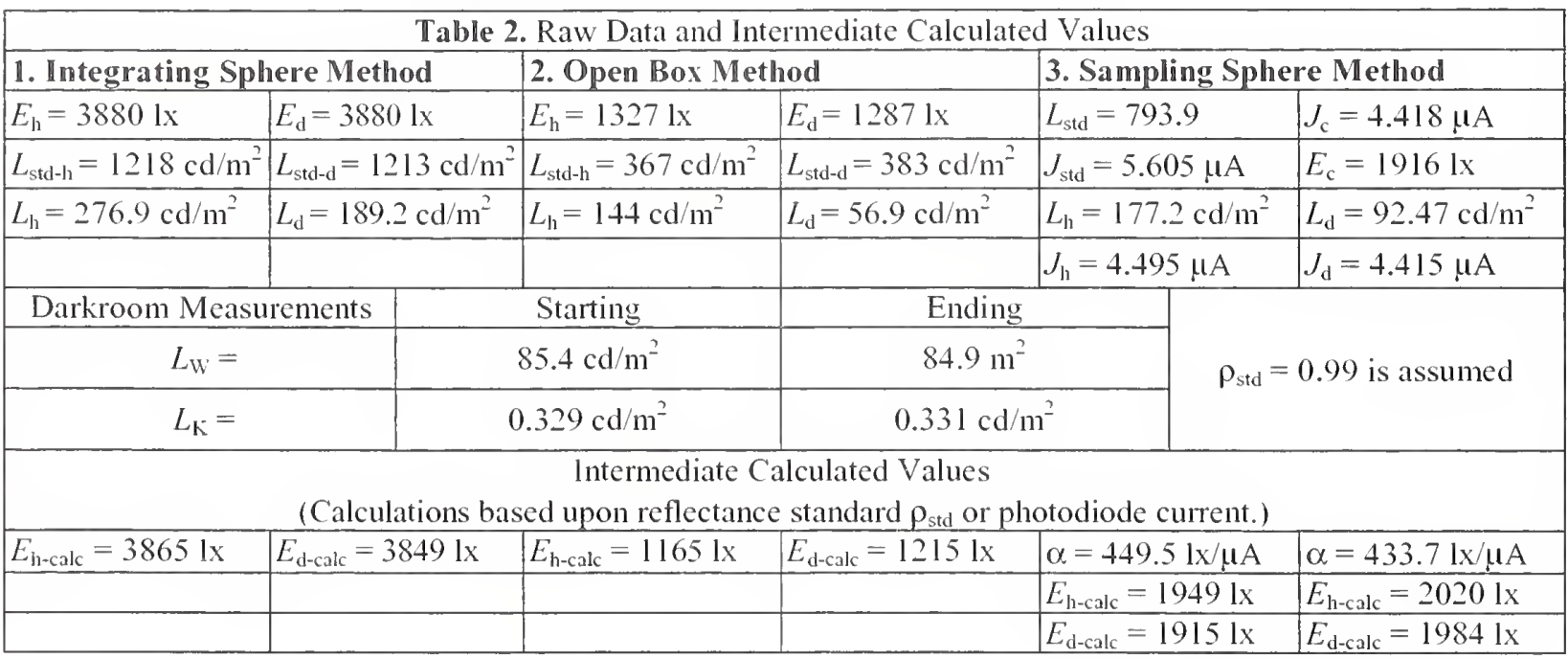

\section{REFERENCES}

1. Flat Panel Display Measurements Standard, Video Electronics Standards Association, Publication FPDM, Version 1.0, Section 308-2 Ambient Contrast Ratio, May 15, 1998.

2. We refer to the diffuse reflectance here in many cases. The diffuse reflectance $\rho_{0 / \mathrm{d}}$ is the same as the luminance factor $\beta_{\mathrm{d} 0}$ according to the Helmholtz reciprocity law-see Reference 3, p 12, below. To be entirely clear we could state that we are measuring the luminance factor $\beta_{\mathrm{d} / 8}$ or $\beta_{\mathrm{d} / 10}$ instead of using the term diffuse reflectance $\rho$; but because there is usually much less than a $1 \%$ error introduced in a measurement of the luminance factor at $\theta=10^{\circ}$ instead of at the normal $\theta=0$, we will not make the distinction here for the sake of simplicity and notation.

3. Absolute Methods for Reflection Measurement, ClE Technical Report, Publication No. CIE44, reprinted 1990. The sampling sphere method used here is similar to the Sharp-Little method in Fig. 10. 

